\title{
Reflets
}

Revue d'intervention sociale et communautaire

\section{Être jeune mère et survivante de violences conjugales en contexte rural et francophone minoritaire : une étude de besoins à Prescott-Russell}

\section{Laurence Clennett-Sirois}

Volume 21, numéro 1, printemps 2015

Violences faites aux femmes et contextes minoritaires

URI : https://id.erudit.org/iderudit/1032549ar

DOI : https://doi.org/10.7202/1032549ar

Aller au sommaire du numéro

Éditeur(s)

Reflets, Revue d'intervention sociale et communautaire

ISSN

1203-4576 (imprimé)

1712-8498 (numérique)

Découvrir la revue

Citer cet article

Clennett-Sirois, L. (2015). Être jeune mère et survivante de violences conjugales en contexte rural et francophone minoritaire : une étude de besoins à Prescott-Russell. Reflets, 21(1), 73-105. https://doi.org/10.7202/1032549ar
Résumé de l'article

Cet article s'appuie sur une étude de besoins réalisée pour Maison Interlude House, dans les comtés unis de Prescott-Russell (Ontario) auprès de six participantes francophones aux prises avec la violence conjugale. Malgré la présence d'organismes dont le mandat est de leur venir en aide, ces jeunes mères monoparentales vivant en milieu semi-rural dans une province à majorité anglophone se trouvent généralement dans une situation de non-choix qui revêt son lot de conséquences. Grâce à leurs récits de vie, aux informations recueillies auprès d'organismes travaillant à l'autonomisation de cette population et à un groupe de discussion visant à identifier des pistes de solutions, nous dressons un portrait des défis rencontrés et des solutions évoquées.
Tous droits réservés @ Reflets, Revue d’intervention sociale et communautaire, 2015
Ce document est protégé par la loi sur le droit d'auteur. L'utilisation des services d'Érudit (y compris la reproduction) est assujettie à sa politique d'utilisation que vous pouvez consulter en ligne. 


\section{Être jeune mère et survivante de violences conjugales en contexte rural et francophone minoritaire : une étude de besoins à Prescott- Russell ${ }^{1}$}

Laurence Clennett-Sirois, Ph D.

Professeure à temps partiel

Université d'Ottawa et Université du Québec en Outaouais

\section{Résumé}

Cet article s'appuie sur une étude de besoins réalisée pour Maison Interlude House, dans les comtés unis de Prescott-Russell (Ontario) auprès de six participantes francophones aux prises avec la violence conjugale. Malgré la présence d'organismes dont le mandat est de leur venir en aide, ces jeunes mères monoparentales vivant en milieu semi-rural dans une province à majorité anglophone se trouvent généralement dans une situation de nonchoix qui revêt son lot de conséquences. Grâce à leurs récits de vie, aux informations recueillies auprès d'organismes travaillant à l'autonomisation de cette population et à un groupe de discussion visant à identifier des pistes de solutions, nous dressons un portrait des défis rencontrés et des solutions évoquées.

Mots clés : Violences conjugales, monoparentalité, minorisation, autonomisation, maternité précoce, récits de vie. 
Drawing on results from a needs analysis conducted for Maison Interlude House, located in the United Counties of PrescottRussell (Ontario), this article sheds light onto the challenges faced by six female Francophone participants, survivors of domestic violence. Despite the work of organisations whose mandate is to support them towards autonomy, these young single mothers, living in a semi-rural area within a mostly Anglophone province, often find themselves in a situation where they have little to no choice. Using data from their life stories, information gathered via various organisations working towards the empowerment of this population and a focus group, we underline the difficulties these women face when trying to escape domestic violence, as well as some of the solutions that were identified.

Key words: Domestic violence, single parenting, minorization, empowerment, early motherhood, life stories.

Cet article tire ses origines d'une analyse de besoins réalisée pour le compte de la Maison Interlude House (MIH) grâce à une recherche-action-participative menée en partenariat avec ses anciennes usagères, ses employées et sa direction. Basée à Hawkesbury dans l'Est ontarien, la MIH est l'une des pionnières de la province en tant que maison d'hébergement et en tant que maison de première étape ${ }^{2}$ offrant des services adaptés aux femmes francophones et à leurs enfants (Coderre, 2014). En 2012, la directrice de la MIH nous exprimait sa préoccupation, laquelle concernait spécifiquement les jeunes mères qu'elle rencontrait dans le cadre de ses fonctions. Pour plusieurs d'entre elles, il s'agissait d'un retour à la maison d'hébergement; elles y avaient séjourné en tant qu'enfants alors que leurs propres mères fuyaient un conjoint et une relation marquée par la violence conjugale. Une explication possible de cette problématique serait le type de services offerts dans le comté de Prescott-Russell où se trouve la MIH. En effet, selon Coderre (2014, p. 54), dans PrescottRussell, « il n'y a aucune ressource de $2^{\mathrm{e}}$ étape. Durant un court séjour, [les femmes] ne peuvent pas rebâtir leur vie. Par la suite, 
"Nous défendons

l'idée selon laquelle malgré la présence d'organismes dont le mandat est de venir en aide à cette population, les participantes rencontrées se retrouvent dans une situation de nonchoix puisque les différents facteurs de risque forment un modèle écologique (Lévesque, 2010) plaçant les jeunes mères monoparentales au sein de rapports de minorisation multiples (Garneau, 2010).» les embuches sont nombreuses pour le faire : peu de ressources facilement accessibles, la pauvreté les guette, peu de possibilités d'emplois, entre autres ".

Afin de mieux comprendre les difficultés rencontrées par les jeunes mères monoparentales francophones qui vivent en situation linguistique minoritaire et qui sont survivantes de violences conjugales, nous avons réalisé en 2012 une étude de besoins. Le présent article présente les principaux résultats tirés de l'analyse combinée de leurs récits de vie, des visites auprès de ressources situées au Québec ainsi que du groupe de discussion réalisé avec les participantes en fin de projet. Nous défendons l'idée selon laquelle malgré la présence d'organismes dont le mandat est de venir en aide à cette population, les participantes rencontrées se retrouvent dans une situation de non-choix puisque les différents facteurs de risque forment un modèle écologique (Lévesque, 2010) plaçant les jeunes mères monoparentales au sein de rapports de minorisation multiples (Garneau, 2010). Ces obstacles rencontrés et ces rapports de minorisation limitent ainsi les possibilités d'échapper durablement aux dynamiques de la violence conjugale. Dans ce qui suit, nous présentons le contexte théorique et empirique de notre recherche, le processus méthodologique ainsi que les résultats obtenus quant aux différentes problématiques perçues. Nous terminons par la présentation de notre principale recommandation, soit le développement, dans un avenir rapproché, d'une ressource adaptée aux besoins des participantes et à la réalité de Prescott-Russell.

\section{Contexte théorique et empirique}

Nous nous intéressons aux jeunes femmes ayant connu une maternité précoce, laquelle peut être comprise comme une grossesse menée à terme et "survenant avant l'âge de 20 ans " (MSSSQ 2004;2008, cité dans Lévesque, 2010,p.198).La littérature identifie de nombreux risques en lien avec la maternité précoce, incluant les difficultés financières associées à une éducation moins 
poussée et conséquemment à des possibilités d'emplois moindres, l'isolement social, les problèmes de santé mentale et le risque accru de vivre une relation de violence conjugale (Boden, Fergusson et Horwood, 2007; Lévesque, 2010). Malgré le fait que la maternité précoce s'éloigne de la "norme procréative " (Bajos et Ferrand, 2006), d'autres recherches suggèrent au contraire que du point de vue de la jeune mère, elle puisse être perçue comme donnant accès à " une relation aimante, une reconnaissance sociale et une reconnaissance de leur identité " (Ali, et collab., 2013, p. 589). La grossesse en jeune âge permet ainsi parfois à ces femmes, particulièrement celles issues d'un milieu défavorisé, d'avoir accès à des services publics (aide à l'emploi et au logement, soutien scolaire) auxquels elles auraient eu difficilement accès en d'autres circonstances (Ali, et collab., 2013, p. 600-601).

Par rapport aux processus pluriels de minorisation, nous faisons référence aux multiples catégories d'identité occupées par les participantes à ce projet, lesquelles structurent et soutiennent des rapports de pouvoir inégaux, les plaçant dans une situation minoritaire. S'appuyant sur les écrits de Colette Guillaumin (1972), Stéphanie Garneau (2010, p. 24) explique :

Le concept de minorité est avant tout sociologique et politique. En effet, le statut de minoritaire ne se réduit pas à une simple différence, ni à une question statistique ou démographique. Il repose plutôt sur une relation sociale qui met en scène un dominant et un dominé [...]. Ainsi, sur la base d'une supposée différence - physique ou culturelle, souvent les deux -, les personnes qui composent un groupe minoritaire sont traitées différemment et de manière inégale par rapport au reste de la population.

À cette notion de minorisation, nous ajoutons les contributions de Crenshaw (2005) sur l'intersectionnalité. Celle-ci problématise les rapports complexes et multiples entre les différentes catégories d'identité (entre autres, le sexe, la classe sociale, la race et l'ethnicité) que nous occupons en tant qu'individus et comme groupes. Ces 
"Nous avançons donc l'idée que les participantes à cette recherche se trouvent en situation de dépossession ${ }^{3}$ de par leurs appartenances à différentes catégories d'identités perçues comme minoritaires (notamment de jeunes, femmes, mères monoparentales, peu scolarisées, francophones, et défavorisées) et puisqu'elles vivent dans un contexte linguistique, politique et démographique minoritaire par rapport à la majorité anglophone en Ontario." rapports structurent des relations de pouvoir, de privilèges et de minorisation qui sont indissociables des contextes dans lesquels ils interviennent et sont articulés. Nous avançons donc l'idée que les participantes à cette recherche se trouvent en situation de dépossession ${ }^{3}$ de par leurs appartenances à différentes catégories d'identités perçues comme minoritaires (notamment de jeunes, femmes, mères monoparentales, peu scolarisées, francophones, et défavorisées) et puisqu'elles vivent dans un contexte linguistique, politique et démographique minoritaire par rapport à la majorité anglophone en Ontario. Cette dépossession complique leurs démarches d'émancipation par rapport aux situations de violences qu'elles ont connues, parfois comme enfants et adolescentes, mais dans tous les cas comme jeunes adultes.

Les recherches - recensées par Lévesque (2010) - qui s'intéressent aux relations marquées par la violence conjugale dans lesquelles les jeunes mères se retrouvent ont déjà démontré la prévalence du phénomène. En effet, en 1997, Convington et collab. (cités dans Lévesque, 2010, p. 198) établissent à 5,4\% le pourcentage de mères ayant connu la maternité au cours de l'adolescence et ayant subi de la violence de la part de leur partenaire. Une décennie plus tard, cette même statistique passe à $67,2 \%$ selon Lindhorst et Oxford (2008, cités dans Lévesque, 2010, p. 198). Toutefois, si ces recherches s'intéressaient toutes aux mères mineures, peu combinaient ces analyses aux autres catégories d'identité minoritaire occupées par leurs participantes. En effet, une seule s'intéressait aux mères adolescentes autochtones, alors qu'une autre s'intéressait aux jeunes mères participant « à un programme de traitement des dépendances " (Lévesque, 2010, p. 199-201). L'exercice de combinaison des théories de l'intersectionnalité et des processus de minorisation nous parait donc une avenue prometteuse, afin de mieux saisir les obstacles rencontrés par les participantes de Prescott-Russell.

Sur le plan socioéconomique, nous remarquons déjà que la situation à Prescott-Russell, selon les données statistiques colligées auprès de Statistiques Canada (2007, pour le recensement de 2006) et d'organismes œuvrant dans la région, est marquée par des difficultés. Particulièrement, nous notons que le revenu médian, 
après impôts, des familles monoparentales où le parent est femme, était de 33550 \$ dans le comté de Prescott-Russell et chutait à 24365 \$ à Hawkesbury. Les familles monoparentales (au nombre de 2 885) étaient d'ailleurs en forte majorité dirigées par une femme (2 160 familles). Le niveau de scolarité des adultes dans la région est également peu élevé, les femmes âgées de 15 et plus comptant pour la majorité des personnes sans diplôme, certificat ou grade (2 050 femmes contre 1635 hommes, sur une population totale de 12267 personnes vivant à Hawkesbury), bien qu'une proportion égale d'hommes et de femmes obtienne un diplôme d'études secondaires. Si au niveau postsecondaire, les diplômées sont plus nombreuses que les diplômés, cela ne se traduit pas de manière équivalente sur le plan de l'employabilité, plus d'hommes que de femmes étant en emploi (à Hawkesbury spécifiquement, $60,7 \%$ des hommes ont un emploi, contre $46,5 \%$ de femmes).

Une agence de services sociaux de la région,VALORIS pour enfants et adultes ${ }^{4}$, nous a communiqué ses données quant aux caractéristiques sociodémographiques des jeunes mères (16 à 30 ans) ayant reçu de leurs services. Ces données offrent un portrait guère plus reluisant. Entre le $1^{\mathrm{er}}$ avril 2008 et le 30 septembre 2012, 47 jeunes mères survivantes de violences conjugales avaient reçu des services deVALORIS. Outre un état civil variable - nous ne disposons pas de ces informations pour 16 femmes, mais parmi les autres, 13 étaient célibataires, 9 séparées, 8 en union de fait et 1 mariée - nous constatons une situation de précarité financière généralisée. En effet, des 24 femmes dont le statut d'emploi était connu, plus de la moitié (14) était sans emploi; sur le plan de la scolarité, des 16 femmes ayant partagé cette information, 2 (âgées de 15 et 17 ans) étaient en cours d'achèvement de leurs études secondaires, 4 avaient au plus leur diplôme d'études secondaires, 9 détenaient un diplôme de formation aux adultes ou permettant de pratiquer un métier et une seule avait un baccalauréat.

Les statistiques colligées par la MIH permettent également de prendre conscience d'une situation préoccupante par rapport à la précarité des femmes de moins de 30 ans qui ont eu recours à leurs services. Pour l'année d'opération 2012-2013, 51 d'entre elles ont bénéficié d'un service à la MIH. Elles avaient pour principale 
"À l'instar d'autres chercheurs (dont Benoit, et collab., 2011; Gurr, et collab., 2008), nous tenons à réitérer que la violence conjugale n'est pas le fait de groupes spécifiques de la société et qu'elle affecte toutes les femmes, sans égard à leur âge ou leur appartenance culturelle, ethnique, de classe sociale ou autre. Nous estimons toutefois que l'interrelation de différents facteurs peut contribuer au développement et à la persistance d'une relation marquée par la violence conjugale, sans toutefois en être son origine. " source de revenus, Ontario au travail ${ }^{5}$, un emploi à temps partiel, ou encore une pension d'invalidité (la MIH ne dispose pas de ces données pour toutes les femmes qui ont transigé par leurs services); pour celles dont le niveau de scolarité était connu, la plupart avaient un diplôme d'études secondaires ou moins. Le niveau de scolarité de ces jeunes femmes ayant été en contact avec la MIH complique également leur intégration au marché du travail, où pour celles (avec ou sans enfant) qui ont fourni ces informations, 8 n'ont pas obtenu leur diplôme d'études secondaires, 5 avaient ce diplôme en main et 2 ont mentionné avec un niveau de scolarité " autre ", sans que nous sachions à quoi cet " autre » niveau réfere.

À l'instar d'autres chercheurs (dont Benoît, et collab., 2011; Gurr, et collab., 2008), nous tenons à réitérer que la violence conjugale n'est pas le fait de groupes spécifiques de la société et qu'elle affecte toutes les femmes, sans égard à leur âge ou leur appartenance culturelle, ethnique, de classe sociale ou autre. Nous estimons toutefois que l'interrelation de différents facteurs peut contribuer au développement et à la persistance d'une relation marquée par la violence conjugale, sans toutefois en être son origine. Pour reprendre les paroles de Lévesque (2010, p. 205) : «La présence d'un facteur de risque, en juxtaposition avec d'autres facteurs de risque, peut favoriser ou précipiter l'occurrence d'une problématique, sans en être la cause. " Les facteurs de précarité soulignés ci-dessus, lesquels ne sont ni la cause ni la source de violence vécue par ces femmes, s'inscrivent dans ce que Lévesque nomme le "modèle écologique " (2010) et compliquent le cheminement de femmes qui sont aux prises avec un conjoint violent. Précisons ici que nous priorisons l'approche des risques et de la résilience en la matière, et ce, tant sur le plan théorique que sémantique. Nous adoptons ainsi une position critique par rapport au "paradigme de la vulnérabilité " en favorisant une référence aux facteurs de risque plutôt qu'aux facteurs de vulnérabilité. En effet, bien que ce paradigme reconnaisse les effets structurels des inégalités entre les sexes et qu'il critique les approches centrées sur l'individu qui ne tiennent pas compte des multiples formes d'oppression et d'inégalités, il masque généralement le pouvoir d'agir et l'agentivité des femmes (Higgins, Hoffman et 
"L'approche des risques et de la résilience permet au contraire la reconnaissance de ce pouvoir d'agir et de cette agentivité, tout en considérant les effets des systèmes d'oppression qui se trouvent au centre de l'analyse intersectionnelle, classisme, racisme on sexisme. »
Dworkin, 2010). L'approche des risques et de la résilience permet au contraire la reconnaissance de ce pouvoir d'agir et de cette agentivité, tout en considérant les effets des systèmes d'oppression qui se trouvent au centre de l'analyse intersectionnelle, classisme, racisme ou sexisme. Cette approche tient aussi compte des conséquences d'expériences vécues, que ce soit sur le plan familial, amoureux, scolaire ou autre (Kulkarni, Kennedy et Lewis, 2010). Par son regard holiste, l'approche des risques et de la résilience est d'ailleurs en cohérence avec notre approche féministe et notre adoption du modèle écologique proposé par Lévesque (2010), lequel permet d'appréhender les obstacles auxquels les femmes sont confrontées.

Dans sa description du modèle écologique, Lévesque (2010) identifie quatre niveaux de facteurs qui interviennent et fonctionnent de concert avec d'autres afin de créer une situation d'autant plus difficile à surmonter. Nous présentons ici les éléments de chaque niveau qui concernent les jeunes mères que nous avons rencontrées. Au niveau individuel, Lévesque souligne les caractéristiques propres à la personne qui font augmenter le risque qu'elle devienne victime de violence, dont la grossesse précoce et les antécédents de violence; le niveau relationnel porte sur les interrelations associées à un risque de violence accru, dont la consommation de narcotiques dans le milieu, l'absence de stabilité conjugale et la présence de violence avant la grossesse; au niveau communautaire, l'environnement (les «cadres de vie ") influence également le risque de violences, ce qui comprend le fait de vivre dans un milieu socioéconomique appauvri, de ne pas occuper un emploi rémunéré en dehors du domicile et l'«absence ou faible présence de soutien social " (Lévesque, 2010, p. 205206). Le quatrième niveau du modèle écologique présenté par Lévesque, le niveau social, rassemble les facteurs s'inscrivant à l'échelle de la société; il concerne, entre autres, les conceptions des rôles propres aux femmes et aux hommes et le partage inégal du pouvoir entre les femmes et les hommes (Lévesque, 2010, p. 206).

À notre avis, la force du modèle écologique permet de ne pas mettre l'accent sur les facteurs individuels (qui, associés aux catégories d'identité occupées par les individus, sont souvent 
évoqués pour expliquer les risques accrus rencontrés par certains groupes), mais de souligner en quoi l'environnement dans lequel évoluent les femmes rencontrées peut également contribuer à leurs difficultés. Ainsi, ce modèle souligne l'importance de tenir compte des contextes afin de comprendre les risques face à la violence conjugale et rejoint en ce sens les contributions des analyses intersectionnelle. Ces dernières notent en effet en quoi les situations de privilège ou d'oppression ne peuvent être entièrement comprises sans référence aux contextes dans lesquels se trouvent les individus (Harper, 2012). Spécifiquement, Lévesque (2010, p. 209) explique :

Pourtant, les facteurs de risque qu'on attribue à l'individu sont souvent liés aux environnements dans lesquels ces derniers vivent, aux opportunités qui leur sont offertes, aux politiques publiques qui encadrent les pratiques et aux normes sociales liées à la violence conjugale et à la maternité précoce. Il en ressort donc une compréhension incomplète et morcelée, parce que décontextualisée.

"...le modèle

écologique nous permet de réfléchir aux conséquences de deux composantes de l'environnement dans lequel vivent les participantes à notre recherche, lesquelles à notre avis sont caractéristiques de la situation linguistique minoritaire ainsi que de la situation semirurale du comté de Prescott-Russell. »
Plus encore, le modèle écologique nous permet de réfléchir aux conséquences de deux composantes de l'environnement dans lequel vivent les participantes à notre recherche, lesquelles à notre avis sont caractéristiques de la situation linguistique minoritaire ainsi que de la situation semi-rurale du comté de Prescott-Russell. Comme nous le développerons davantage au cours des prochaines pages, le fait de vivre en français dans une province à majorité anglophone - bien que dans une localité majoritairement francophone - où les services en français ne sont pas adéquatement développés et financés limite les possibilités pour ces femmes d'échapper à une relation violente (Crenshaw, 2005). Par exemple, plusieurs contraintes pèsent sur celles qui désirent augmenter leur sécurité financière par le biais de l'emploi; plusieurs employeurs requièrent un niveau d'études plus élevé et un bilinguisme de la part de leurs employés, ce qui n'est pas nécessairement le cas des participantes que nous avons rencontrées. 
"L'adoption de ce modèle, à ne pas confondre avec le paradigme écologique présenté par Damant, Paquet et Bélanger (2001), rejoint selon nous les orientations privilégiées par de nombreux groupes féministes qui "refusent de découper la réalité des femmes en mille et une problématiques isolées les unes des autres "..."
Elles doivent aussi souvent être en mesure de se déplacer en voiture puisqu'aucun service de transport en commun n'est disponible dans Prescott-Russell. Notons également que le manque de services en français pour femmes victimes et survivantes de violences conjugales en Ontario limite chez elles la possibilité de déménager ailleurs dans la province. L'adoption de ce modèle, à ne pas confondre avec le paradigme écologique présenté par Damant, Paquet et Bélanger (2001), rejoint selon nous les orientations privilégiées par de nombreux groupes féministes qui « refusent de découper la réalité des femmes en mille et une problématiques isolées les unes des autres"(Boisclair, et collab., 2010, p. 215). En effet, Damant, Paquet et Bélanger (2001) proposent une comparaison des paradigmes de l'empowerment que sont les perspectives technocratique, structurelle et écologique. Elles soulignent en quoi ce dernier reconnait les effets des structures sur le vécu des individus, mais ne cherche pas une transformation des rapports inégalitaires, mais plutôt "à en diminuer l'impact négatif» (Damant, Paquet et Bélanger, 2001, p. 139). Notre propre démarche, qui reconnait la complexité des vécus et les effets des structures sociales, laisse toutefois place, comme souligné ci-dessus, à la résilience des individus et à leur capacité d'action, ce qui permet d'entrevoir la possibilité de contestation et de transformation de ces structures.

Ayant présenté notre emploi du modèle écologique, comme cadre théorique de référence permettant d'envisager la complexité des vécus et des obstacles rencontrés par les participantes, nous nous penchons à présent sur la démarche méthodologique adoptée pour ce projet. Cette démarche et l'analyse qui l'accompagne permettent la création d'un espace pour l'expression des participantes, la validation de leurs vécus et expériences, ainsi que leur contribution à un projet visant une transformation sociale. Nous détaillons, dans ce qui suit, les visées d'autonomisation et de prise de parole ainsi que les orientations éthiques spécifiques qui ont influencé nos choix méthodologiques. 


\section{Travailler ensemble : le processus méthodologique}

Afin de mieux comprendre les problématiques rencontrées par les jeunes mères qui quittent une relation marquée par la violence conjugale, nous avons réalisé des entrevues sous forme de récits de vie avec six jeunes mères qui avaient été en contact avec la MIH (4 d'entre elles ont bénéficié de l'hébergement et 2 de services externes) et qui ont été recrutées par des employées de l'organisme. Les récits de vie sont associés à de nombreux avantages, notamment dans le cadre d'une recherche féministe auprès de jeunes mères, puisqu'ils permettent de "dresser un portrait plus complet de leurs expériences " (Lévesque, 2010, p. 212) et d'établir des relations plus égalitaires et moins objectifiantes entre les personnes impliquées, soit la chercheuse embauchée par la MIH et les jeunes mères (Montell, 1998). Considérant que Statistique Canada révèle que moins de $30 \%$ des victimes de violences conjugales font appel aux services d'une maison d'hébergement (2011, p. 14), nous avons également tenté de recruter des femmes qui n'auraient pas transité par la MIH en apposant des affiches dans les locaux de différents organismes communautaires de la région. Cette méthode de recrutement n'a toutefois pas été concluante et peut constituer une première limite de notre recherche; nous ne sommes pas parvenues à obtenir des informations auprès de femmes qui auraient choisi de ne pas faire appel aux services de la MIH pour diverses raisons, tels les préjugés ou une méconnaissance par rapport à l'offre de services ou l'approche qui y est privilégiée.

De par les origines de ce projet et les mesures mises en place lors de sa réalisation, nous nous inscrivons dans une approche de recherche-action-participative, laquelle vise à " accompagner le changement » et adopte des approches de recherche permettant "de valoriser un processus de production des connaissances réalisé de concert avec les acteurs concernés " (Anadón et Couture, 2007, p. 3). Particulièrement, les approches participatives, dont la recherche-action, conservent un ancrage dans la communauté auprès de laquelle les chercheuses sont investies et structurent 
"Au centre de ces choix méthodologiques résidait notre volonté de placer l'expérience et le vécu des participantes au coeur de notre démarche, comme le préconise la recherche féministe..." leur méthodologie de sorte à permettre un engagement de la communauté concernée. En effet, les approches participatives " envisagent le sujet (la personne ou la communauté) dans son contexte et tentent de comprendre la signification et les implications du problème de recherche et de sa solution pour la communauté " (Anadón et Couture, 2007, p. 3). Ainsi, les savoirs et expertises de la direction et des employées de la MIH ont été mobilisés à toutes les étapes de la réalisation du projet (Morris et Muzychka, 2002), notamment lors de la réflexion entourant la collecte des données, la définition de la problématique, le recrutement des participantes, la réalisation de certaines étapes de collecte de données, leur analyse et la rédaction du rapport final. À la suite de nos rencontres auprès de l'équipe de travail à la MIH et de notre appropriation de la littérature, nous avons développé des outils pour la cueillette de données auprès des jeunes mères. Ces instruments, soit un calendrier des parcours de vie (Gaudet, 2002) et un guide d'entrevue semi-dirigée permettant de couvrir certains thèmes, ont appuyé la réalisation des récits de vie. Divisé en différentes sections (situation actuelle, enfance, adolescence, période adulte et perspectives futures), le guide permettait d'aborder les défis et les réussites sur le plan des différents parcours (scolaire, familial, amoureux, professionnel, etc.) et les sources d'aide (sur le plan du réseau personnel, des ressources communautaires, publiques ou institutionnelles). Au centre de ces choix méthodologiques résidait notre volonté de placer l'expérience et le vécu des participantes au cœur de notre démarche, comme le préconise la recherche féministe (Ollivier et Tremblay, 2000). La durée des entrevues individuelle variait d'une à trois heures. Les participantes aux récits de vie ont eu accès à la transcription de leurs récits personnels et ont par la suite participé à un groupe de discussion, d'une durée d'un peu plus de deux heures. Cette étape de réflexion collective a été précédée d'une discussion des résultats préliminaires avec des membres de l'équipe de la MIH. Elle a permis d'approfondir les principales difficultés rencontrées, de discuter des résultats obtenus par la recherche, d'en approfondir leur analyse et d'imaginer le développement d'une ressource qui serait spécifique aux jeunes mères survivantes 
de violences conjugales. Ce processus de mise en commun des analyses initiales et du développement de réflexions par rapport aux suites à donner au projet est central à la recherche-action. Selon Kurtzman (2009, p. 95), à cette étape, "[1]es textes ne sont pas encore rédigés dans un langage accessible, mais c'est par rapport à ces premiers écrits que le groupe doit évaluer si le travail des chercheuses semble respectueux de son action, et s'il se reconnait dans la façon dont elles traduisent les résultats de la recherche-action".

L'intérêt d'impliquer les jeunes mères dans l'analyse des résultats des entrevues et dans la réflexion quant à des pistes de solution envisageables, en plus des membres de l'équipe de la $\mathrm{MIH}$, est double. Cela permet en effet de valider les résultats mis de l'avant, mais aussi de contribuer au renforcement des capacités d'action et au développement d'habiletés chez les participantes (Levac, 2013). Cette implication des participantes est fréquente en recherche féministe, laquelle « valorise [...] le fait de travailler ensemble à partir de questions que les femmes jugent problématiques, d'abord à les comprendre, puis à agir " (Ollivier et Tremblay, 2000, p. 39). Par ailleurs, les entretiens de groupe revêtent un intérêt certain dans le cadre de recherches comme la nôtre, puisque cette méthode, comme le soulignent Baribeau et Germain (2010, p. 33), peut être employée « dans la résolution de problèmes qui visent l'amélioration de situations en rechercheaction ou en recherche-développement pour faciliter les analyses de besoins ". Rappelons que notre projet visait effectivement une meilleure compréhension des besoins des jeunes mères survivantes de violences conjugales ainsi que l'amorce d'une réflexion quant à une ressource qui pourrait mieux répondre à leurs besoins. Précisions enfin que plusieurs idées relatives à cette ressource imaginée ont été tirées de rencontres auprès de responsables d'organismes à Québec et Montréal qui offrent leurs services à cette population. Ces rencontres, qui visaient à réfléchir aux possibilités de services et sources de financement envisageables, ont été réalisées de concert avec la directrice de la MIH, la chercheuse principale et des responsables de deux organismes québécois, soit Mères et Monde (Québec) et Mères avec pouvoir (Montréal). 
Les entrevues individuelles avec les jeunes mères et l'entretien de groupe ont eu lieu dans les bureaux de la MIH à Hawkesbury (Ontario).Trois motifs ont influencé ce choix de lieu pour la tenue de ces entrevues. D'abord, nous souhaitions introduire l'organisme aux femmes qui ne l'auraient peut-être pas connu avant l'entrevue, dans l'éventualité où le recrutement externe aurait fonctionné, en leur donnant l'occasion d'en connaitre l'adresse et d'en visiter les locaux. Ensuite, la MIH est un lieu sécuritaire et à accès contrôlé, offrant ainsi une mesure de protection additionnelle aux personnes impliquées, particulièrement si leur sécurité était toujours menacée par un ex-conjoint. Ce lieu nous donnait aussi accès à un bureau privé, ce qui permettait de réaliser l'entrevue en toute confidentialité. Enfin, l'accès à une intervenante en tout temps pendant la durée des entrevues individuelles et de l'entrevue de groupe était également essentiel compte tenu de la nature sensible des sujets qui pouvaient être abordés lors des récits de vie. En collaboration avec l'équipe de la MIH, nous avons aussi convenu de mesures permettant d'assurer que les participantes ne soient pas confrontées à des obstacles financiers qui empêcheraient leur participation à notre recherche. Nous avons donc pris des dispositions pour couvrir les frais de garde d'enfants et de déplacements. Des collations étaient offertes aux participantes et elles ont toutes reçu un montant de $65 \$$ en guise de dédommagement pour l'entrevue individuelle. Ce montant n'était pas annoncé au moment du recrutement pour ne pas

"... l'analyse des récits de vie visait à identifier " la signification exacte $d u$ message ", du point de vue de celles qui formulent ces messages et en considérant «ce qui est dit [...], tel quel, directement et ouvertement "..." influencer indument la participation de femmes qui auraient pu être mal à l'aise pendant l'entrevue, mais qui seraient restées pour obtenir cette compensation. La somme était offerte aux participantes, dans une enveloppe, vers la fin de l'entrevue. La directrice de la MIH estimait qu'il s'agissait là d'une autre manière de reconnaitre l'importante contribution de ces femmes au projet et aussi de les remercier pour le temps qu'elles nous avaient accordé.

Réalisée au moyen d'une analyse de contenu manifeste, l'analyse des récits de vie visait à identifier « la signification exacte du message ", du point de vue de celles qui formulent ces messages et en considérant « ce qui est dit [...], tel quel, directement et ouvertement "(L'Écuyer, 1990, p. 14; 22). Nous avons donc 
procédé en suivant librement les six étapes du modèle général d'analyse de contenu développé par L'Écuyer (1990) et nous avons catégorisé les données manuellement en suivant le modèle mixte. Ce modèle se base sur les catégories préexistantes qui sont tirées de la littérature, particulièrement par rapport aux facteurs de risques documentés, et des catégories induites du matériel, soit celles qui n'étaient pas prévues par la littérature (L'Écuyer 1990, p. 66). Comme évoquées ci-dessous, les analyses ont été validées par les participantes et certains membres du personnel de la MIH lors des rencontres suivant la réalisation des récits de vie, permettant ainsi la mise en place d'un processus dialogique.

À la suite de l'analyse des récits de vie des participantes, de l'exploration des ressources existantes (notamment les visites des ressources Mères et monde et Mères avec pouvoir) et de la rencontre sous forme de groupe de discussion avec les participantes aux récits de vie, nous constatons la nécessité d'interventions par le biais du développement de ressources pour soutenir ces femmes. Comme détaillé dans la prochaine section, ces jeunes mères, déjà avant d'entrer dans la relation abusive et encore à la suite de leur séparation, sont confrontées à des facteurs de risque accrus qui les placent dans des rapports de minorisation pluriels et qui minent leurs efforts pour en sortir.

\section{La réalité rencontrée : les multiples facteurs de risque qui interviennent dans la vie des participantes}

Le partage des récits de vie des participantes et l'analyse commune lors de la discussion de groupe ont fait émerger des facteurs de risque aux niveaux personnel et environnemental, lesquels ont eu des impacts dans la vie des femmes rencontrées. Nous présentons, à tour de rôle, ces différents éléments.

\section{Facteurs de risque personnels}

Les facteurs de risque se situant au niveau individuel et répertoriés à la suite de l'analyse des récits de vie comprennent une vie 
"En effet, le fait

d'avoir connu, ou non, de la violence et de l'instabilité pendant leur vie a été évoqué par toutes les participantes. " (enfance, adolescence et âge adulte) marquée par la violence et l'instabilité, la maternité précoce et les difficultés à l'école (performance et fréquentation).

Le premier facteur associé à des problématiques présentes dans le milieu familial pendant l'enfance, l'adolescence et la vie adulte a marqué le parcours de l'ensemble des participantes. En effet, le fait d'avoir connu, ou non, de la violence et de l'instabilité pendant leur vie a été évoqué par toutes les participantes. Dans une majorité de cas, il s'agissait d'une dynamique familiale connue à laquelle elles ont cherché à échapper. Pour une participante, une telle dynamique était totalement inconnue; les signes avantcoureurs étaient pour elle difficilement identifiables, retardant sa prise de conscience quant à la dynamique de violence qui s'installait.

Ainsi, certaines participantes ont évoqué des violences verbales, psychologiques et physiques de la part de parents comme étant un facteur ayant précipité une mise en ménage avec un partenaire. Ce dernier, aussi violent, les avait initialement charmées et leur offrait la possibilité de se distancer de leurs familles d'origine. Plus encore, la littérature établit un lien entre le choix d'une maternité précoce chez certaines jeunes femmes issues de milieux familiaux à problématiques multiples et la possibilité d'acquérir une indépendance ou une reconnaissance sociale accrue (Ali, et collab., 2013). Une participante à notre recherche, détaillant sa première grossesse à seize ans, en parlait d'ailleurs comme d'une grossesse désirée, perçue comme étant " une porte de sortie " (participante Aurélie ${ }^{6}$ ), même si elle a plutôt choisi de parler d'une grossesse " accidentelle " à ses parents. Pour d'autres, ayant séjourné dans plusieurs foyers d'accueil lorsqu'elles étaient mineures, la mise en ménage semblait être une source de stabilité et d'amour qu'elles n'avaient pas connue antérieurement (participantes Coralie et Dorothée). Pour une participante (Béatrice), au contraire, la dynamique de la violence conjugale était un phénomène totalement étranger et, de son point de vue, aurait contribué au fait qu'elle n'avait pas reconnu certains comportements comme étant abusifs. 
La maternité précoce, choisie ou pas, est un deuxième facteur ayant chamboulé des plans quant à la poursuite des études (trois participantes sur cinq ont estimé qu'elles auraient souhaité obtenir un diplôme ou une certification plus poussée, ce qui a été compliqué du fait de devenir mères — la sixième participante avait effectué un retour aux études au moment de nos rencontres). La maternité en bas âge a aussi des répercussions sur le développement personnel; une participante a souligné qu'elle n'était pas prête et n'avait pas la maturité requise pour devenir mère lorsqu'elle a donné naissance à son premier enfant. Bien que nous reconnaissions que d'autres chercheurs (par exemple, Ali, et collab., 2013) notent les effets positifs pouvant découler d'une grossesse en bas âge, entre autres, la mise en contact avec des organismes susceptibles d'appuyer une femme afin qu'elle échappe à la pauvreté, le développement d'une identité personnelle positive, il semble que de tels effets n'aient pas été ressentis par les participantes.

Enfin, le troisième facteur individuel qui a émergé de notre recherche et qui peut être associé aux deux premiers est celui du

"Ainsi, une scolarité incomplète ou jugée insuffisante serait un marqueur $d u$ processus de minorisation par rapport à la classe sociale qui place les participantes dans un rapport inégalitaire et dans une situation de désavantage économique. " cheminement scolaire. Autant les difficultés scolaires peuvent être des conséquences des problèmes vécus dans la famille d'origine ou de la grossesse précoce, autant elles peuvent constituer un obstacle additionnel à surmonter lorsque les jeunes mères souhaitent échapper à une situation marquée par de la violence à leur égard. Le manque de reconnaissance et de compétences associé à l'absence de diplôme aux niveaux secondaire ou postsecondaire pose en effet un défi additionnel lorsque les participantes souhaitent s'insérer en emploi et améliorer leurs conditions matérielles. Ainsi, une scolarité incomplète ou jugée insuffisante serait un marqueur du processus de minorisation par rapport à la classe sociale qui place les participantes dans un rapport inégalitaire et dans une situation de désavantage économique. 


\section{Facteurs associés à la relation abusive}

Les relations dans lesquelles les participantes étaient impliquées étaient marquées, certes, par la violence conjugale sous toutes ses formes, entre autres, psychologique, verbale, sexuelle, physique et financière. D'autres problématiques sont aussi identifiées dans les récits des participantes, rendant à leur avis la relation - et le fait de quitter cette relation - encore plus complexe. Tout aussi reliés au niveau individuel du modèle proposé par Lévesque (2010), ces facteurs peuvent également être regroupés en trois principales catégories : 1 . les comportements considérés comme illégaux ou illicites; 2 . la précarité financière; 3 . les problèmes de santé (physique ou mentale). Notons que si ces difficultés sont ici présentées sous trois catégories différentes, cela ne signifie pas qu'elles soient indissociables les unes des autres; elles peuvent donner lieu à un système où les difficultés vécues dans une sphère en engendrent d'autres dans une autre sphère. Par exemple, les problèmes de santé mentale peuvent être situés en aval ou en amont des comportements considérés comme illégaux ou illicites, lesquels peuvent se répercuter sur les difficultés financières, ou vice-versa.

En ce qui concerne la première catégorie, celle des comportements considérés comme illégaux ou illicites, notons d'abord que les conjoints de certaines participantes, autant que certaines participantes elles-mêmes, ont connu des démêlés avec la justice pour avoir consommé ou vendu des narcotiques, perpétré des agressions contre la personne ou commis des vols. Les données recueillies auprès de responsables d'organismes œuvrant auprès de cette clientèle (Appartements Augustine-Gonzalez, Maison Pauline-Bonin, Mères avec pouvoir, Mères et monde) corroborent ces résultats. Ces informations illustrent les conséquences de la consommation problématique d'alcool ou de narcotiques, de l'implication dans la prostitution et de la fréquentation de réseaux criminels comme autant de difficultés auxquelles les jeunes mères monoparentales étaient régulièrement confrontées. 
"... la précarité financière a affecté l'ensemble des participantes et représente la deuxième catégorie de difficultés rencontrées au niveau individuel. »
«Tant lors de la cohabitation avec leur ex-conjoint violent qu'à la suite de leur séparation, plusieurs participantes ont dévoilé avoir eu à composer avec des enjeux de santé, tant physique que mentale. »
Les difficultés financières étaient dans certains cas une conséquence directe des comportements illicites, notamment la consommation d'alcool ou de narcotiques. Notons que la précarité financière peut aussi être à l'origine de certains comportements illégaux ou perçus comme illicites, telle la prostitution.

Vécue pendant l'enfance, au moment de la relation avec le conjoint violent et souvent à la suite de la séparation, la précarité financière a affecté l'ensemble des participantes et représente la deuxième catégorie de difficultés rencontrées au niveau individuel. Cette précarité a été décrite de plusieurs manières, soit par des références à des faillites personnelles (Béatrice et Flavie), ou encore par la nécessité de faire appel à l'aide sociale (Coralie, Dorothée et Éléonore), au dépannage alimentaire (Aurélie, Coralie, Dorothée et Flavie) et à du logement subventionné (Aurélie, Coralie, Dorothée, Éléonore et Flavie). Outre ces réalités, les participantes étaient très critiques des allocations de l'aide sociale qui ne tiennent pas compte des couts de logement qui varient selon la ville ou la région où elles habitent, ni des dépenses ponctuelles, telles la rentrée scolaire des enfants. Dans bien des cas, ces mères avaient aussi de la difficulté à obtenir un soutien financier de la part des ex-conjoints, pères de leurs enfants. En effet, certaines étaient en attente d'un jugement par rapport à ces pensions alimentaires, alors que d'autres jugeaient préférable de s'en passer pour ne pas que cela serve de prétexte à plus de contrôle de la part de leurs ex-conjoints. Cette réalité est préoccupante pour deux raisons : les conséquences que peut entraîner la pauvreté dans la vie des femmes et de leurs enfants et l'insécurité financière qui influence souvent la décision de quitter ou non un conjoint violent (Barnett, 2000).

Tant lors de la cohabitation avec leur ex-conjoint violent qu'à la suite de leur séparation, plusieurs participantes ont dévoilé avoir eu à composer avec des enjeux de santé, tant physique que mentale. Cette troisième catégorie de difficultés associées au niveau individuel a été confirmée lors du groupe de discussion, où les participantes ont toutes évoqué des difficultés en lien avec leur santé mentale, notamment les crises d'angoisse et la dépression. Certaines étaient toujours médicamentées, alors 
que d'autres faisaient appel à des spécialistes tant au privé qu'au communautaire. Les intervenantes des organismes rencontrées soulignaient un problème similaire, notant que les problèmes de santé étaient récurrents, entre autres, maux de dos, rhumes et grippes à répétition ou dépression. Selon une intervenante de Mères avec pouvoir, les problèmes de santé seraient de plus en plus fréquents chez cette population :

Intervenante : Il y a autre chose, aussi, que l'on rencontre de plus en plus. C'est... Elles sont malades, nos mères. Malades...

Chercheuse : Sur le plan de la santé mentale et physique?

Intervenante : Physique et mentale, oui. Dépression... Des maux de dos comme ça ne se peut pas. [...] Tu sais, il me semble qu'avant, les jeunes comme ça, il n'en avait pas autant. Mais maintenant... Nos mères ont de gros maux de dos. Le stress, beaucoup, beaucoup de stress... Elles ne sont pas bien. Je ne les trouve pas bien! Fragiles. Tu sais, les rhumes à répétition, les grippes...

Ces problématiques ont potentiellement été exacerbées du fait que les participantes, notamment lors de leur union avec leur partenaire violent, et bien souvent à la suite de leur séparation, étaient généralement isolées et n'avaient donc pas de réseau auquel faire appel pour se sentir appuyées. Ainsi, le sentiment d'être isolées, notamment chez Béatrice et Coralie, a eu des répercussions sur leur santé mentale ainsi que sur les démarches entreprises pour échapper à la relation violente. Dans le premier cas, un partenaire violent a poussé Béatrice à briser les liens avec sa famille et ses amis, ce qui a eu pour effet de l'isoler tant pendant la relation qu'à la séparation, alors qu'elle devait rebâtir les ponts avec les gens de son réseau. Quant à Coralie, elle a vécu un isolement géographique en quittant l'Est ontarien pour emménager avec son conjoint en Outaouais québécois. Le manque de réseau et de 
"... ces problématiques ont pu être exacerbées aussi par le fait que bien souvent les participantes ne connaissent pas leurs droits, en particulier ceux qui se rapportent aux dispositions légales sur la garde des enfants ou sur le logement en tant que locataires. " ressources financières lui permettant de revenir à Hawkesbury a apporté son lot de difficultés à cette participante qui a notamment vécu une bonne partie de sa deuxième grossesse loin de son réseau social et qui se sentait peu appuyée lorsque confrontée à la violence de son conjoint.

Enfin, ces problématiques ont pu être exacerbées aussi par le fait que bien souvent les participantes ne connaissent pas leurs droits, en particulier ceux qui se rapportent aux dispositions légales sur la garde des enfants ou sur le logement en tant que locataires. Cette méconnaissance place les participantes en relation de dépendance et de vulnérabilité face aux avocats, lesquels font leur éducation juridique, mais souvent de manière incomplète. Conséquemment, les participantes se sentent fréquemment non appuyées — sinon tout simplement trahies — par le système judiciaire. Certaines se disaient effectivement mal préparées pour l'audience en cour, d'autres se voyaient contraintes de faire appel à des amis pour comprendre la documentation à remplir, alors que d'autres encore en venaient à perdre confiance envers le système et les forces de l'ordre qui ne les avaient pas adéquatement appuyées quand elles ont eu besoin d'aide.

Les difficultés engendrées par ces facteurs personnels et celles associées à la relation abusive prennent une signification particulière lorsqu' analysées à la lumière de l'environnement dans lequel demeurent les participantes, ce qui est l'objet de la dernière section de cette analyse.

\section{Facteurs associés à l'environnement : vivre en milieu rural et linguistique minoritaire}

La dernière série de facteurs dont il est question dans cet article résulte d'une microanalyse du point de vue des participantes sur les services disponibles dans la communauté, réalisée en parallèle avec une macroanalyse du contexte linguistique minoritaire et rural dans laquelle elles vivent et où ces services sont offerts. Nous débutons avec un survol des expériences évoquées par 
rapport aux principales ressources publiques et communautaires offertes dans la région, dont la MIH de façon spécifique, mais aussi d'autres services mobilisés, ceux de sécurité publique et de justice, les services d'intervention auprès des familles et de lutte contre les violences, les services de soutien au logement, les services de dépannage alimentaire et autres services financiers. Nous enchainons enfin avec une analyse du contexte linguistique et rural dans lequel cette recherche a été réalisée.

Nous l'avons évoqué brièvement, les participantes offrent un portrait mitigé des services auxquels elles ont eu accès lorsqu'elles cherchaient à échapper à la violence de leur ex-conjoint. Notons cependant qu'elles ont offert une appréciation positive de ceux reçus à la MIH, laquelle offre l'ensemble de ses services (ressources externes ou hébergement) dans les deux langues officielles et offre également un accompagnement lorsque les femmes doivent se reloger ou faire appel à différentes ressources dans la communauté.

C'est habituellement lorsqu'elles font appel à d'autres services, en aval ou en amont de leur contact avec des intervenantes de la $\mathrm{MIH}$, que les participantes rencontrent des obstacles. Ceuxci sont associés à des préjugés et à une attitude négative à leur endroit, en fonction des processus de minorisation évoqués cidessus, à leur situation linguistique minoritaire ou encore au fait de vivre dans un milieu rural. En effet, les préjugés et attitudes défavorables ont compliqué l'accès de participantes aux services des forces de l'ordre, lesquelles ont refusé de leur venir en aide sous divers motifs. Dans un cas, la situation dénoncée a été jugée non urgente. Dans un autre cas, lors d'un appel de détresse, une participante a dû rompre subitement la communication et elle était dans l'incapacité de répondre au téléphone par la suite : on a mis beaucoup de temps à venir sur place lui porter secours. Les participantes ont reçu de la part des forces de l'ordre un service qui minait leur confiance à l'égard du système. Par exemple, une mère bénéficiant des services d'aide à l'enfance a eu du mal à dénoncer son ancien conjoint qui contrevenait à une ordonnance de non-contact. Une autre a eu droit à une intervention très minimaliste lorsqu'elle a réclamé de l'aide pour récupérer ses biens dans le logement qu'elle partageait jusqu'à récemment avec son 
"Les facteurs qui pourraient expliquer ces difficultés lors des interactions avec les forces de l'ordre sont les préjugés et attitudes défavorables envers les jeunes femmes, les mères en situation de monoparentalité et l'absence de capital culturel et social (Bourdieu, 1986) permettant aux participantes de faire valoir leurs droits efficacement. " ex-conjoint. Les facteurs qui pourraient expliquer ces difficultés lors des interactions avec les forces de l'ordre sont les préjugés et attitudes défavorables envers les jeunes femmes, les mères en situation de monoparentalité et l'absence de capital culturel et social (Bourdieu, 1986) permettant aux participantes de faire valoir leurs droits efficacement.

Les organismes qui ont pour mandat d'intervenir pour aider les familles et lutter contre la violence, dont les services d'aide à l'enfance, ont fait l'objet d'une appréciation mitigée, ne nous permettant pas ici d'établir de tendance manifeste. Tantôt les participantes louangeaient les services qu'elles y avaient reçus, tantôt elles dénonçaient un manque d'attention de leur part. Le principal reproche consistait en une méconnaissance ou en une non-reconnaissance de leurs besoins. Une participante qualifiait d'envahissante et de trop insistante l'intervention des services d'aide à l'enfance. Une autre estimait qu'une intervenante consultée dans un organisme différent était plus préoccupée par sa manucure que par ce qu'elle lui racontait. Ces attitudes condescendantes et indifférentes pourraient-elles être interprétées à la lumière des processus de minorisation (monoparentalité, faible niveau de scolarité, activités passées associées au crime organisé) qui alimentent les préjugés face aux participantes et se répercutent sur les attitudes à leur endroit?

Les services de soutien au logement et les services d'aide financière sont essentiels à ces jeunes mères confrontées à la précarité financière. Nos données indiquent en effet qu'une majorité a dû faire appel à une forme ou à une autre d'appui au logement ou de soutien financier. Bien qu'Aurélie et Flavie ne soient pas restées longtemps dans un logement subventionné, au moment de l'entrevue, Coralie occupait une habitation à loyer modique alors que Dorothée et Éléonore étaient sur une liste d'attente à cet effet. Pour les femmes ayant séjourné à la MIH, précisons que l'accès à un logement subventionné a été facilité par une disposition ontarienne donnant priorité aux personnes victimes de violences conjugales - cette disposition s'applique aussi aux autres victimes d'abus que peuvent être les personnes âgées et les enfants. Cela est un gain indéniable des luttes menées 
«La combinaison de revenus insuffisants aux couts de logement souvent trop élevés par rapport à ces revenus signifie que les participantes doivent parfois faire appel à des services de dépannage, dont les services d'aide en sécurité alimentaire. " par le mouvement féministe, et plus spécifiquement par les maisons d'hébergement. Cette mesure permet à ces femmes qui souvent ont un faible revenu de ne pas consacrer l'essentiel de leurs ressources financières au logement ou encore d'avoir accès à un logement abordable et de qualité, deux caractéristiques qui sont souvent mutuellement exclusives dans le marché locatif. Selon Houle (2003, p. 39), s'appuyant sur des données recueillies par McAll (1995), « les femmes sur l'assistance sociale pouvaient octroyer entre 60 à $70 \%$ de leurs prestations d'aide sociale au logement. Il ne leur reste plus qu'environ $30 \%$ de leur revenu pour se nourrir, se vêtir et payer les autres dépenses de la vie quotidienne ». Ainsi, si ce n'était de la disposition évoquée cidessus, plusieurs des participantes risqueraient de se trouver dans une situation de sans-abrisme ${ }^{7}$ et de vulnérabilité par rapport au logement. L'accès à un revenu décent est une difficulté rencontrée par les participantes à notre projet, lesquelles dépendaient de l'aide sociale pour subvenir à leurs besoins (trois participantes), sur le revenu de leur conjoint actuel, combiné à leurs revenus d'emploi à temps partiel (deux participantes) ou encore sur un seul salaire (une participante occupait un emploi à temps plein, ayant sa propre garderie en milieu familial). Les difficultés financières associées à "des prestations d'aide sociale inférieures au seuil de faible revenu " (Convergence, 2006, p. 8), à une dépendance envers le revenu du nouveau conjoint, et à une source de revenus tirée d'un travail autonome - lequel est aussi une source de précarité, particulièrement lorsque ce sont des femmes qui occupent ce type d'emploi (Commission du droit de l'Ontario, 2012) — sont le lot de l'ensemble des participantes que nous avons rencontrées et peuvent engendrer une discrimination sur le marché locatif.

La combinaison de revenus insuffisants aux couts de logement souvent trop élevés par rapport à ces revenus signifie que les participantes doivent parfois faire appel à des services de dépannage, dont les services d'aide en sécurité alimentaire. Au moment des entrevues, le service de la Banque alimentaire basée à Hawkesbury connaissait depuis quelque temps des difficultés qui ont mené à des politiques limitant l'accès aux dépannages, entre autres, par l'instauration d'une limite annuelle de cinq dépannages 
par famille et des frais de cinq dollars par visite individuelle et de dix dollars par famille (Blais-Gauthier, 2013; Radio-Canada, 2013). En plus de ces restrictions, problématiques en soi, les participantes ont également évoqué la qualité discutable de certains produits pour lesquels elles avaient payé ainsi qu'une attitude peu courtoise de la part de bénévoles y œuvrant et qui disaient aux usagers qu'ils n'étaient pas les «bienvenus trop souvent $»^{8}$.

Enfin, d'autres intervenants sont venus en aide aux participantes lorsqu'elles étaient aux prises avec leur conjoint violent, ou encore avec des difficultés financières à la suite de leur séparation. Particulièrement, soulignons le travail d'enseignantes qui ont agi pour faire cesser le harcèlement d'un ex-conjoint envers une participante et qui en ont aidé une autre à obtenir des services adaptés pour son enfant, alors que ni le père de l'enfant ni les grands-parents maternels ne croyaient à la validité d'une telle démarche. Des collègues de classe ont aussi préparé un panier de Noël pour venir en aide à une participante, alors que des membres de la communauté religieuse à Hawkesbury ont apporté un appui matériel (achat d'électroménager) et émotif à deux participantes.

Ce que l'ensemble de ces données révèle, lorsqu'analysé à la lumière des résultats de Barnett (2001) sur l'importance du soutien social et communautaire, ainsi que des contributions théoriques sur les processus de minorisation (Garneau, 2010; Guillaumin, 1972), de l'intersectionnalité (Crenshaw, 2005) et du modèle écologique de Lévesque (2010) est l'interconnexion et la complexité des défis rencontrés par les participantes. Ces défis sont nombreux et complexes, se situant tant avant qu'elles aient rencontré le conjoint violent (exposition à la violence ou directement victime de violence au sein de la famille d'origine; difficultés scolaires; problèmes de consommation), qu'au moment de leur relation (violences multiples, grossesses précoces, précarité financière, problèmes de consommation et autres comportements jugés illicites) ou à la suite de la séparation (harcèlement et violence qui se poursuivent, isolement et précarité financière). À ces défis, d'autres s'ajoutent, associés ceux-là à la situation de minorité linguistique et de semi-ruralité dans lesquelles vivent les participantes. 
"Cet accès limité

à des services en français restreint la possibilité pour les femmes de l'Est ontarien de quitter la région pour s'éloigner de leur ex-conjoint. Notons que cet accès limité à des services en français affecte également l'éducation des enfants qui pourraient avoir de la difficulté à poursuivre leur scolarité dans leur langue si leurs mères choisissaient de s'établir à l'extérieur de l'Est ontarien."
Nous l'avons évoqué précédemment, des recherches démontrent qu'à l'échelle de la province, les ressources francophones pour femmes survivantes de violences conjugales sont insuffisantes : « Et partout au Canada en contexte minoritaire francophone, on parle de carence en terme de services accessibles en français pour les femmes et leurs enfants vivant une situation de violence » (FNFCF, 2005 , p. 1). Cet accès limité à des services en français restreint la possibilité pour les femmes de l'Est ontarien de quitter la région pour s'éloigner de leur ex-conjoint. Notons que cet accès limité à des services en français affecte également l'éducation des enfants qui pourraient avoir de la difficulté à poursuivre leur scolarité dans leur langue si leurs mères choisissaient de s'établir à l'extérieur de l'Est ontarien.

À ce manque de services en français s'ajoutent les problématiques associées au contexte semi-rural qui caractérise beaucoup de municipalités dans cette région. Particulièrement, l'accès difficile à certains services à cause de l'absence de transport en commun, comme l'ont souligné les participantes lors du groupe de discussion, est caractéristique de cette région où les habitants doivent souvent se fier aux services de taxi faute de posséder une voiture. D'autres problématiques repérées par Coderre (2014) dans le cadre de sa recherche consistent en un manque relatif d'anonymat, à la difficulté d'assurer une sécurité financière en se trouvant un emploi après la séparation sans avoir à quitter la région, les pressions familiales et communautaires pour régler ces histoires « en famille».

\section{Discussion des résultats : une situation sans issue?}

Ce que ce portrait révèle est que les participantes rencontrées dans l'Est ontarien se retrouvent à l'intersection de rapports de pouvoir multiples, notamment parce qu'elles sont minorisées en tant que femmes, jeunes, mères monoparentales, personnes peu scolarisées, ayant parfois un passé associé à des comportements illicites. Cette situation de minorisation, associée à des caractéristiques dites 
"Leur mauvaise connaissance de l'anglais les empêche non seulement de se renseigner sur les refuges, mais aussi, trop souvent, de bénéficier de la sécurité qu'ils sont sensés procurer. Certains de ces établissements invoquent en effet le manque de ressources ou de personnel bilingue pour justifier leur refus d'accueillir les femmes ne parlant pas anglais. "
" individuelles " selon le modèle proposé par Lévesque (2010), les exclut d'un accès ${ }^{9}$ et d'un traitement égalitaire lorsqu'elles font appel à différents services pour se soustraire à la violence conjugale. Nos résultats rejoignent en ce sens les propos de Levac (2013) qui souligne que pour plusieurs jeunes mères, le portrait type en est un marqué par de nombreuses difficultés : pauvreté, exclusion sociale, problèmes de santé. Les problématiques associées à leur situation géographique et à leur appartenance linguistique minoritaire viennent quant à elles compliquer leurs démarches d'émancipation. Effectivement, ces deux dernières caractéristiques engendrent des difficultés structurelles qui limitent la mobilité des femmes qui souvent n'ont pas accès au transport permettant l'obtention de certains services, ou encore n'auraient pas accès à des services en français si elles décidaient de vivre ailleurs en Ontario. Ces deux problématiques pourraient d'ailleurs être ajoutées au modèle écologique suggéré par Lévesque (2010) et refléter la réalité des femmes francophones en Ontario qui ne vivent pas en milieu urbain.

Ainsi, nous ajoutons le fait de faire partie d'un groupe linguistique minoritaire au niveau communautaire présenté par Lévesque (2010), puisque cela signifie que les services et les possibilités d'améliorer les conditions de vie sont souvent limités. À ce sujet, soulignons que Crenshaw (2005, p. 58), discutant du contexte étasunien en lien avec l'intersectionnalité, identifie également la difficulté additionnelle associée au fait de ne pas bien maitriser l'anglais :

La barrière de la langue constitue un autre obstacle structurel qui empêche les femmes non anglophones de tirer parti des aides existantes (Banales, 1990, p.E5).Leur mauvaise connaissance de l'anglais les empêche non seulement de se renseigner sur les refuges, mais aussi, trop souvent, de bénéficier de la sécurité qu'ils sont sensés procurer. Certains de ces établissements invoquent en effet le manque de ressources ou de personnel bilingue pour justifier leur refus d'accueillir les femmes ne parlant pas anglais. 
Nous y ajoutons également la situation de semi-ruralité, aussi caractéristique du niveau communautaire, qui accroit l'isolement, complique les démarches et compromet l'anonymat des femmes qui font appel à des ressources d'aide pour lutter contre la violence conjugale.

\section{Conclusion}

Pour terminer, soulignons que les interactions entre les différents niveaux du modèle écologique de Lévesque (2010) permettent de saisir le caractère omniprésent des processus de minorisation qui affectent les participantes. Celles-ci se retrouvent effectivement, et souvent depuis leur enfance, dans une situation de non-choix où elles précipitent par exemple une mise en ménage pour échapper à des violences dans leur famille d'origine, ou choisissent la maternité à un âge précoce comme symbole de leur émancipation familiale ou encore doivent vivre avec les conséquences liées au fait de demeurer dans l'Est ontarien après avoir quitté un conjoint violent (Coderre, 2014) ou de vivre dans l'isolement (social et linguistique) si elles choisissent de s'établir ailleurs en Ontario.

La principale limite de notre projet de recherche est propre à l'échantillon et au recrutement de participantes, puisque nous n'avons pas été en mesure, comme souligné ci-dessus, de rencontrer des femmes qui n'auraient pas fait appel aux services de la MIH en tant qu'adultes. Étant donné que nombre de femmes quittent effectivement un conjoint violent sans pourtant faire appel aux services d'une ressource d'hébergement (pour des raisons diverses comme la possibilité de recourir à du soutien informel de la part d'amis, de membres de la famille ou d'autres personnes, ou encore comme les préjugés quant à l'offre de services et à l'approche préconisée en maison d'hébergement), il aurait été intéressant de parler à certaines d'entre elles. Ces rencontres auraient permis de connaitre tant les obstacles qu'elles ont rencontrés que les ressources auxquelles elles ont pu faire appel, produisant ainsi une compréhension plus approfondie des 
"Les approches préconisées par ces organismes reconnaissent autant la complexité des vécus des femmes et les effets des systèmes d'oppression multiples que le pouvoir d'agir des femmes qui fréquentent leurs ressources. " effets des rapports de minorisation qui touchent les jeunes mères survivantes de violences conjugales et qui vivent en milieu semirural et linguistique minoritaire. Ainsi, des recherches futures pourraient miser sur le recrutement de jeunes mères ayant quitté un partenaire violent sans toutefois avoir fait appel à des ressources pour femmes victimes de violences conjugales.

Néanmoins, à la lumière de l'analyse de besoins que nous avons réalisée pour la $\mathrm{MIH}$, nous avons recommandé le développement d'une ressource propre aux jeunes mères monoparentales, survivantes de violences conjugales. Cette ressource pourrait s'inspirer des approches adoptées à Mères avec pouvoir et Mères et monde, lesquelles misent sur le développement et la consolidation de capacités d'action et reconnaissent le potentiel de leadership, pour reprendre l'expression de Levac (2013), des jeunes mères en dépit des nombreux obstacles qu'elles rencontrent. Les approches préconisées par ces organismes reconnaissent autant la complexité des vécus des femmes et les effets des systèmes d'oppression multiples que le pouvoir d'agir des femmes qui fréquentent leurs ressources. Ainsi, sur le plan tant pratique que théorique, leur travail rejoint la perspective que nous avons adoptée dans ce projet. Toutefois, le travail permettant d'endiguer les facteurs qui contribuent à la minorisation des jeunes femmes aux niveaux communautaire et social requiert aussi une transformation en profondeur des normes et pratiques culturelles ancrées dans notre société. Il semble en effet que ce soit des facteurs de risque au niveau social (Lévesque 2010), dont les croyances et les attitudes négatives à l'égard des participantes en situation de minorisation à cause de leur appartenance de sexe, de leur classe sociale, de leur âge et de leur situation géographique et linguistique, qui engendreraient et soutiendraient ces situations parsemées d'obstacles et qui nécessitent donc une intervention à plus grande échelle. D'ici là, il est possible de rêver à une ressource spécifique, comme l'ont fait les participantes à cette recherche, les membres de l'équipe de la MIH et les responsables consultées auprès des organismes. Cette ressource permettra entretemps d'outiller les jeunes mères, notamment en leur donnant accès à un logement sécuritaire et abordable, à un service de garde, 
à des possibilités de développement personnel, académique et professionnel, afin de mieux répondre aux défis rencontrés aux niveaux individuels et relationnels. Plus encore, en s'inspirant des approches préconisées par les organismes visités, cette ressource pourra être un espace d'autonomisation et de développement d'une prise de conscience collective par et pour les femmes qui $\mathrm{y}$ transigeront et y contribueront.

\section{Notes}

1 L'auteure souhaite remercier les participantes qui ont partagé leurs récits de vie et ont participé au groupe de discussion; les équipes des différentes ressources basées au Québec — particulièrement Mères et Monde et Mères avec pouvoir — pour leur temps et conseils; les membres du personnel de Maison Interlude House (particulièrement Céline Pelletier, directrice) pour leur appui tout au long de ce projet; ainsi que les personnes suivantes, pour leurs commentaires sur des versions préliminaires de cet article : Bonnie Diamond, Ghislaine Sirois et Céline Pelletier. Les suggestions des deux évaluatrices anonymes et des responsables de ce numéro de Reflets nous ont également grandement aidée.

2 Nous reprenons la définition qu'en offre Coderre (2014, p. 54), soit que ce type d'hébergement vise à " offrir un séjour adapté aux besoins de chaque femme, mais en moyenne entre 3 à 4 semaines ».

3 Nous utilisons " dépossession " comme traduction de disempowerment, à la suggestion faite par Oristelle Bonis qui a traduit le texte, originalement en anglais, de Crenshaw (2005).

4 VALORIS pour enfants et adultes offre une panoplie de services sociaux à la population de Prescott-Russell, agissant à titre de Société de l'aide à l'enfance, offrant des services aux témoins et survivants de violences conjugales, ainsi que des services aux personnes ayant des difficultés sur le plan de la santé mentale, de la déficience intellectuelle et du développement psychomoteur (VALORIS, n.d.).

5 Ontario au travail est le programme d'aide sociale en Ontario. Il comporte une aide financière provisoire et un programme d'aide à l'emploi pour les Ontariennes et Ontariens admissibles, permettant de couvrir les frais de logement et de nourriture. Les prestations d'aide sociale varient en fonction de différents facteurs, tels la taille de la famille, le revenu, le cout du logement et les avoirs des requérants (Ministère des services sociaux et communautaires, 2012).

6 Afin de préserver l'anonymat des participantes, nous avons remplacé leurs prénoms par les pseudonymes suivants : Aurélie, Béatrice, Coralie, Dorothée, Éléonore et Flavie - en plus de modifier certains passages des transcriptions qui pourraient permettre de les identifier.

7 À l'instar de Benoît et collab. (2011,p. 2), nous considérons le sans-abrisme comme ne signifiant pas que les femmes vivent nécessairement dans la rue : elles peuvent être hébergées temporairement chez " une amie, un membre de la famille ou dans un logement fourni par une agence de bienfaisance ", tout en demeurant vulnérables du point de vue de leur logement. 
8 Notons que depuis la collecte de données, la Banque alimentaire de Hawkesbury a modifié ses pratiques à la suite des changements survenus au sein du Conseil d'administration de l'organisme.

9 Notons que cette question d'accès est parfois tributaire d'une auto-exclusion, comme dans le cas de la participante Éléonore qui a choisi de ne pas faire appel aux services d'hébergement de la MIH par crainte que sa venue, avec un bébé, ne dérange les autres femmes qui s'y trouvaient déjà.

\section{Bibliographie}

ALI, Diana S., et collab. (2013). «'I Became a Mom': Identity Changes in Mothers Receiving Public Assistance ", Journal of Social Service Research,Vol. 39, № 5, p. 587-605.

ANADÓN, Marta, et Christine COUTURE (2007). «La recherche participative, une préoccupation toujours vivace ", sous la direction de Marta Anadón, La recherche participative : multiples regards, Québec, Presses de l'Université du Québec, p. 1-10.

BAJOS, Nathalie, et Michèle FERRAND (2006). "L'interruption volontaire de grossesse et la recomposition de la norme procréative ", Sociétés contemporaines, $N^{\circ}$ 61, p. 91-117.

BARIBEAU, Colette, et Mélanie GERMAIN (2010). "L'entretien de groupe : considérations théoriques et méthodologiques ", Recherches qualitatives, Vol. 21, N¹, p. 28-49.

BARNETT, Ola W. (2000). «Why battered women do not leave, Part 1. External inhibiting factors within society ", Trauma, Violence \& Abuse, Vol. 1, N4, p. 343-372.

BARNETT, Ola W. (2001). "Why battered women do not leave, Part 2. External inhibiting — Social support and internal inhibiting factors ", Trauma, Violence \& Abuse, Vol. 2, N¹, p. 3-35.

BENOÎT, Monique, et collab. (2011). La vulnérabilité du logement des femmes francophones monoparentales, sans-abri ou sans domicile fixe. Une étude des besoins sur la situation à Sudbury, Présentation faite dans le cadre du $4^{\mathrm{e}}$ colloque international des programmes locaux et régionaux de santé, Gatineau, 27-30 juin 2011, http://www.plrs-ottawa2011.com/pdf_liste/BENOIT_monique_E5.pdf

BLAIS-GAUTHIER, Samuel (2013). «La banque alimentaire de Hawkesbury confirme le changement de ses règles ", LeDroit, édition du 9 mars 2013, http://www.lapresse.ca/le-droit/201303/09/014629432-la-banque-alimentaire-de-hawkesbury-confirme-le-changement-de-ses-regles.php

BODEN, Joseph M., David M. FER GUSSON et L. John HORWOOD (2007). « Early motherhood and subsequent life outcomes ", The Journal of Child Psychology and Psychiatry, Vol. 49, No 2, p. 151-160.

BOISCLAIR, Odile, et collab. (2010). "L'intervention féministe dans les centre de femmes : en action pour un monde plus juste! ", sous la direction de Corbeil, Christine et Isabelle Marchand, L'intervention féministe d'hier à aujourd'hui. Portrait d'une pratique sociale diversifiée. Montréal : les éditions du remue-ménage, p. 211-227.

BOURDIEU, Pierre (1986). "The forms of capital ", dans John G. Richardson (dir.), Handbook of theory and research for the sociology of education, New York, Westport (Connecticut), London, Greenwood Press, p. 241-258.

CODERRE, Cécile, avec COALITION CONTRE LA VIOLENCE FAITE AUX FEMMES DE PRESCOTT-RUSSELL (2014). « Portrait régional des violences faites aux femmes dans 
les comtés unis de Prescott Russell et des services », réf. du 6 juin 2014, 196 pages, réf. du 14 août 2014, http://coalitionviolencepr.ca/images/client/documents/Documents_pertinents/ Rapport_final_-_14mai_2014.pdf

COMMISSION DU DROIT DE L'ONTARIO (2012). "Les travailleurs vulnérables et travail précaire - Rapport préliminaire », Toronto, Commission du droit de l'Ontario, 198 pages, réf. du 26 septembre 2014, http://www.lco-cdo.org/vulnerable-workers-interim-report-fr.pdf

CONVERGENCE, COOPÉRATIVE D'EXPERTES-CONSEILS (2006). Étude de besoins en matière de services d'hébergement pour les femmes francophones et leurs enfants fuyant une relation violente dans la région de Toronto, Rapport présenté à l'Action ontarienne contre la violence faite aux femmes, Ottawa, $82 \mathrm{p}$.

CRENSHAW, Kimberlé Williams (2005). «Cartographie des marges : intersectionnalité, politique de l'identité et violences contre les femmes de couleur ", [traduction Oristelle Bonis], Cahiers du genre, $\mathrm{N}^{\circ} 39 / 2$, p. 51-82.

DAMANT, Dominique, Judith PAQUET et Jo BÉLANGER (2001). «Recension critique des écrits sur l'empowerment ou quand l'expérience de femmes victimes de violence conjugale fertilise des constructions conceptuelles ", Recherches féministes, Vol. 14, N², p. 133-154.

FÉDÉRATION NATIONALE DES FEMMES CANADIENNES-FRANÇAISES (FNFCF) (mars 2005). «La violence faite aux femmes et les femmes francophones en contexte minoritaire : un dossier prioritaire de la FNFCF ", Les Brèves,Vol. 8, № 1, p. 1-2, réf. du 14 août 2014, http://franco.ca/affc/documents/Les_Breves_mars_05.pdf

GARNEAU, Stéphanie (2010). "Penser le pluralisme des francophonies minoritaires canadiennes : de la logique identitaire à la question sociale ", Reflets, revue d intervention sociale et communautaire, Vol. 16, No 2, p. 22-56.

GAUDET, Stéphanie (2002). Responsabilité et socialisation au cours du passage a l'âge adulte. Le cas de jeunes adultes de la région montréalaise, [thèse de doctorat], Montréal, Université du Québec à Montréal et INRS-Centre Urbanisation, culture et société, 313 p.

GUILLAUMIN, Colette (1972). Idéologie raciste, genèse et langage actuel, Paris, La Haye, Mouton, 247 p.

GURR, Jane, et collab. (2008). Défaire les liens entre la pauvreté et la violence faite aux femmes. Un guide de ressources, Ottawa, Agence de la santé publique du Canada, 39 p., réf. du 22 janvier 2012, http:// www.phac-aspc.gc.ca/ncfv-cnivf/pdfs/fem-brklnks-fra.pdf,

HARPER, Élizabeth, et collab. (2012). Regards sur l'intersectionnalité, CRI-VIFF, Montréal, 26 p., réf. du 20 décembre 2014, http://www.relais-femmes.qc.ca/files/CRI-VIFF-Regards_sur_intersectionnalite.pdf

HIGGINS, Jenny A., Susie HOFFMAN et Shari L. DWORKIN (2010). "Rethinking gender, heterosexual men, and women's vulnerability to HIV/AIDS ", American Journal of Public Health, Vol. 100, N³, p. 435-445.

HOULE, Pascale (2003). "Les obstacles à l'intégration au marché du travail des femmes monoparentales à faible revenu ", Reflets, revue d'intervention sociale et communautaire, Vol. 9, $\mathrm{N}^{\circ}$ 2, p. 34-57.

KULKARNI, Shanti J.,Angie C. KENNEDY et Carol M. LEWIS (2010). «Using a risk and resilience framework and feminist theory to guide social work interventions with adolescent mothers ", 
Families in Society : The Journal of Contemporary Social Service, Vol. 91, N³, p.217-224.

KURTZMAN, Lyne (2009). "Les enjeux éthiques de la recherche-action féministe : une réflexion critique sur les règles et pratiques de recherche ", dans Fatou Sow, (dire.), La recherche féministe francophone. Langue, identités et enjeux, Paris, Karthala, p. 91-99.

L'ÉCUYER, René (1990). Méthodologie de l'analyse développementale de contenu : méthode GPS et concept de soi, Sillery, Presses de l'Université du Québec, 502 p.

LEVAC, Leah (2013). «'Is this for real?' Participatory research, intersectionality, and the development of leader and collective efficacy with young mothers ", Action Research,Vol.11, N4, p. 423-441.

LÉVESQUE, Sylvie (2010). «Accoucher à 16 ans, sans violence : une réalité possible? État empirique de la situation et réflexion critique ", Santé mentale au Québec,Vol. 25, No 1, p. 195-219.

MÈRES AVEC POUVOIR (2013). réf. du 26 juillet 2012, http://www.mapmontreal.org.

MÈRES ET MONDE (2011). réf. du 26 juillet 2012, http://www.meresetmonde.qc.ca.

MINISTÈRE DES SERVICES SOCIAUX ET COMMUNAUTAIRES (2012), "Ontario au travail ", réf. du 29 mai 2013, http://www.mcss.gov.on.ca/fr/mcss/programs/social/ow/

MONTELL, Frances (1998). "Focus group interviews: A new feminist method ", NWSA Journal, Vol. 11, No 1, p. 44-71.

MORRIS, Marika, et Martha MUZYCHKA (2002), La recherche-action participative. Un outil pour le changement social!, Ottawa, ICREF/CRIAW, 78 p.

OLLIVIER, Michèle, et Manon TREMBLAY (2000), Questionnements féministes et méthodologie de la recherche, Paris, L'Harmattan, $256 \mathrm{p}$.

RADIO-CANADA (2013). "La Banque alimentaire de Hawkesbury met fin à la controverse ", [émission du 2 avril 2013], réf. du 2 mai 2013, http://www.radio-canada.ca/regions/ ottawa/2013/04/22/007-banque-alimentaire-hawkesbury-controverse-fin.shtml

STATISTIQUE CANADA (2011). "La violence familiale au Canada : Un profil statistique ", réf. du 22 mars 2013, http://www.statcan.gc.ca/pub/85-224-x/85-224-x2010000-fra.htm

STATISTIQUE CANADA (2007). "Prescott and Russell, Ontario (Code3502) (tableau). Profils des communautés de 2006, Recensement de 2006, produit $n^{\circ}$ 92-591-XWF au catalogue de Statistique Canada ", réf. du 22 mars 2013, http://www12.statcan.ca/census-recensement/2006/ dp-pd/prof/92-591/index.cfm?Lang=F

VALORIS (2011), réf. du 3 novembre 2012, http://www.valorispr.ca/en/ 